\title{
Effect of Scapular Kinesiotaping as an Adjunct to Dynamic Neuromuscular Stabilization Exercises on Upper Extremity Functions in Stroke Patients
}

\author{
Rutuja Jadhav ${ }^{1}$, Shamla Pazare ${ }^{2}$ \\ ${ }^{1}$ Assistant Professor, St. Andrews College of Physiotherapy, Pune, Maharashtra, India. \\ ${ }^{2}$ HOD and Professor, Chaitanya Medical Foundation's College of Physiotherapy, Pune, Maharashtra, India. \\ Corresponding Author: Rutuja Jadhav
}

DOI: https://doi.org/10.52403/ijhsr.20220103

\begin{abstract}
Background and Purpose: Stroke is one of the leading causes of death and disability in India. Upper extremities get more affected in Middle cerebral artery syndrome. Stroke patients have lack of functional ability of upper extremity due to scapular malalignment resulting from paralysis of the scapular muscles. Kinesiotape helps to realign the muscles and thus it becomes easy to activate or inhibit them. We hypothesised that Dynamic Neuromuscular Stabilisation exercises along with kinesiotape could improve upper extremity function.

Methodology: Ethical clearance was obtained. Informed consent was taken. 30 patients were selected according to inclusion and exclusion criteria. 15 subjects were randomly divided into experimental and control group. Subjects were given Dynamic neuromuscular stabilisation exercises for scapular strengthening. The experimental group was given Kinesiotaping to the scapula using muscle technique. Pre and post treatment assessment was done using Upper Extremity Function Index, grip strength and scapular abduction position with MB ruler.

Result: The intergroup comparison revealed a highly significant difference for upper extremity function index and grip strength whereas scapular abduction was not significant.

Conclusion: The Scapular Kinesiotaping as an adjunct to Dynamic Neuromuscular Stabilization Exercises is significantly effective to improve upper extremity functions and grip strength in post stroke patients.
\end{abstract}

Keywords: Stroke, Upper Extremity Functions, Dynamic Neuromuscular Stabilization Exercises, Scapular Kinesiotaping.

\section{INTRODUCTION}

Stroke is the sudden loss of neurological function caused by an interruption of the blood flow to the brain.[1] Stroke is one of the leading causes of death and disability in India. The incidence rate is $119-145 / 100,000$ based on the recent population-based studies. 2 The estimated prevalence rate of stroke range, $84-262 / 100,000$ in rural and 334424/100,000 in urban areas.[2] The incidence of stroke is about 1.25 times greater for males than females.[1]
Stroke is a clinical syndrome divided into two broad categories; Ischemic stroke and Haemorrhagic stroke. 1) Ischemic stroke is the most common type, affecting about 80 per cent of individuals with stroke, and results of a thrombus, embolism or conditions that produce low systemic perfusion pressure, causing lack of cerebral blood flow. As a result the brain is deprived of needed oxygen and glucose, which in turn disrupts cellular metabolism, and leads to injury and death of tissues.[1] 2) Haemorrhagic stroke that occurs when 
blood vessels rupture, causing leakage of blood in or around the brain.[1]

The most common site of stroke is Occlusion of Middle cerebral artery. It is one of the two main branches of the internal carotid artery and supplies entire lateral aspect of cerebral hemisphere and subcortical structures including the internal capsule (posterior portion), corona radiate, globus pallidus (outer part) and the putamen, most of the caudate nucleus.[1] Atherosclerosis is the main reason of stroke, which is characterized by formation of plaques. It shows accumulation of substances like lipids, fibrins on arterial walls that lead to progressive narrowing of blood vessels. This results in ischemic stroke which is caused due to reduction in the cerebral blood flow to particular area.[1] Stroke is characterized by paralysis or weakness of one side of the body opposite the side of the lesion. Patients diagnosed with a stroke often present with a combination of muscle weakness or muscle imbalance, decreased postural control, muscle spasticity, poor voluntary control and body malalignment. Middle cerebral artery syndrome shows more involvement of upper extremities. The functional impairment of the upper limb is a usual consequence of a stroke that affects about $80 \%$ of stroke survivors.[3]

Upper extremities play an important role in carrying activities of daily living such as clothing, bathing, grooming, eating, writing, cooking etc. They also play a role in reaching and manipulations which require adequate control in upper limb muscles as well as hand. Scapula plays a major role in upper limb functioning. A good scapular position is necessary to perform manipulations and other hand activities, as a component of stabilization support for the distal movements. If there is an improper position of the scapula, then the muscles either go into shortening or lengthening which reduces the efficiency of those muscles as stabilizers thus making them less active and reduces their capacity to maintain ideal scapular position in order to have efficient upper extremity functions and hand manipulations.[4] Stroke affects the tone in scapular muscles making them weak and inactive hence alters the position of scapula. Therefore, an upper extremity biomechanics change with a change in muscle forces generated by scapular muscles, which affects the proximal stability of upper extremity that deteriorates the upper limb functioning and affects the quality of life of patient.[5] In stroke rehabilitation program, regaining normal voluntary movement and functional use of upper extremity is a very challenging for patients as well as therapists.

During the flaccid stage, the scapula descends from its normal horizontal level, causing the scapula to rotate downward as the trunk tends to lean or shorten towards the hemiplegic side.[6] Kibler (2003) stated that muscle imbalance or weakness, scapular muscle fatigue may lead to an altered glenohumeral proprioception, muscular inhibition, impaired co-ordination and timing of movements are the causes for scapular dyskinesis.[6] Improper alignment and instability of scapula on chest wall will significantly affect the available active as well as passive range of motion of shoulder joint. These all above problems lead to reduction of patient's ability to use the arm functionally. This results in difficulty in performing the activities of daily living and reduction in the quality of motor performance. Also, these residual impairments and functional limitations are the major causes of social restrictions in stroke patient. So, for proper functioning of upper extremity, it is necessary to orient the scapula to its normal resting position.

There have been many studies regarding rehabilitation interventions to improve hand functions, but still upper limb disability is the topic of research. Regaining mobility in the upper extremities is often more difficult than in lower extremities which impacts the progress of rehabilitation. There are many interventions used to improve upper extremity functions such as Electromyography Biofeedback, functional 
electrical stimulation (FES)[7], constrained induced movement therapy[8], Robotic devices for movement therapy[9], virtual reality training, and bimanual activities taskspecific training.

Stroke Patients have paralysis of the scapular muscles which results in scapular malalignment which lead to lack of functional ability of upper extremity[10] In stroke patients the proximal stability for the upper extremities is disturbed as the position of the scapula is changed due to muscle imbalance such as scapula is elevated, protracted and abducted.[10] This biomechanical inefficiency of scapula alters the upper extremity functions, which affects patient's quality of life. It is necessary to correct the scapular muscle imbalance to have proper functioning of upper limb. Muscle imbalance results from hyperactivity of upper trapezius and inactivity of serratus anterior and a rhomboids muscle.[11] Due to presence of proximal muscle weakness; there is reduction in the strength of distal muscles. The handling abilities of the stroke patients are reduced due to decreased grip strength. Many interventions have been practiced for scapular stabilization to improve upper extremity performance.

Dynamic

Stabilization exercises principles of developmental kinesiology and the neurophysiological aspects of a maturing postural locomotor system. DNSE is a rehabilitation approach which stimulates movement control centres in the brain to activate how our bodies were meant to move by restoring and stabilizing locomotor function. This approach compares the individual's stabilizing pattern with the stabilization developmental pattern of a healthy infant. The treatment approach emphasizes training of natural posturallocomotion patterns as defined by developmental kinesiology. According to this concept, in order to get a stabilization pattern which resembles those of new-borns, brain must be stimulated in appropriate and optimum positions. This can be achieved by the activation of the stabilizers (mainly scapula) by placing the patient in developmental positions along with activities of extremities.[12] Research has shown Dynamic neuromuscular stabilization exercises being beneficial to improve the strength of the stabilizers and thus can improve the upper extremity functions.[12] As proximal stability is pre-requisite for distal mobility, stabilization exercises for proximal limb muscles has become an important exercise regimen in rehabilitation of upper extremity.[12] This concept says that to have a proper stabilization, the proximal parts of body like core and scapular muscles need to be activated and maintained in optimum position on which the distal function depends. Dynamic Neuromuscular Stabilization Exercises uses the approach in which every purposeful movement is reinforced by the co-ordinated activity of the segmental postural stability.[12] The goal of DNSE is to achieve optimal muscle co-ordination by exercising in developmental positions. So we can achieve activation and stabilization of proximal parts by assuming various positions and then by giving simple task to affected and unaffected side. It brings the supporting joints and segments into a functionally aligned position.[12]

A study conducted on sports persons is found to be effective in core activation and the patients performance was improved post this intervention.[13] A similar study carried out by Alena Kobesova et. al. showed that Shoulder girdle exercises based on DNS may generate clinically significant gains in hand muscle strength.[13]

On the other hand, Kinesiotaping is a treatment method used in conjunction with other therapeutic techniques in the treatment of various musculoskeletal and neuromuscular deficits. Kinesiotaping may be one of the techniques to gain the symmetry of scapula at the scapulothoracic joint and to correct muscle imbalance (hyperactivity of upper trapezius and inactivity of serratus anterior and rhomboids muscles).[11,14] It also aims to give free 
range of motion in order to allow the body's muscular system to heal itself biomechanically.[15] Kinesiotaping can be used as an adjunct during the rehabilitation program to enhance functional recovery of an upper extremity by reducing pain, improving scapular alignment, supporting the muscle hence improving muscle contraction in weak muscles, reduces muscle fatigue and stimulating or inhibiting muscle function, overcoming muscle weakness and improving proprioceptive function of the joint structure. It provides feedback to the muscles to maintain preferred postural alignment.[11, 14, 15] Yasukawa studied the results of the Kinesiotaping application in improving upper extremity control and function in the acute paediatric rehabilitation setting.[16] Based on a clinical evaluation, Kinesiotaping was applied to facilitate a functional upright position of the trunk, to assist with positioning the shoulder in neutral alignment, and to provide palmar stability and arch support for the involved hand. He concludes that the use of the Kinesiotaping method appeared to have improved purposeful movement and provided needed stability of the shoulder and hand.[16] Yasukawa et al. found that the application of the Kinesiotaping provided the proper body alignment to allow performance of reach, grasp, and release and manipulation tasks.[16]

So it is hypothesized that it will stabilize the scapula and thus improving the functional expectancy of the upper extremity by inhibiting the hyperactivity of the upper trapezius and activating the serratus anterior and rhomboids muscles.[11] So, we state that kinesiotaping along with DNSE will improve scapular alignment and facilitate faster improvement of the strength. Hence this study will be carried out to check the added effect of kinesiotaping along with DNSE on upper limb function and the grip strength.

So it was hypothesized that it will improve the functional expectancy of the upper extremity by inhibiting the hyperactivity of the upper trapezius and activating the serratus anterior and rhomboids muscles which results in stabilizing the scapula.[6] Hence this study will be carried out to check the added effect of kinesiotaping along with DNSE on upper limb function and the grip strength.

\section{MATERIALS AND METHODOLOGY}

Kinesiotapes, stickers, scissor, aneroid manometer for grip strength, camera for photographs and MB ruler software were the materials used in the study.

On obtaining ethical clearance from research ethics committee, the participants were screened and written informed consent was taken.

Using purposive sampling, 30 male and female patients in the age group 40-65 years, having single episode of MCA stroke within last 6 months with Brunnstrom's voluntary control grading for hand $\geq 3$ stages were involved in the study. Those having cognitive deficit (MOCA score <26), respiratory, cardiovascular or neurological disorder that could interfere in participation of exercise program, incomprehensive patients and patients who are hypersensitive to kinesiotape were excluded. After screening, the subjects were randomly divided by chit randomization method into 2 groups, experimental group and control group, 15 subjects with mean age $(50.8+6.25$ and $51.8+6.47)$ included in each group respectively.

Baseline assessment of all the subjects was done according to Upper Extremity Function Index, grip strength and scapular deviation. Experimental group received scapular Kinesio taping to trapezius, rhomboids and serratus anterior muscles. Kinesiotape was kept for 3 days and next tape was applied after a gap of 3 days. Both groups $\mathrm{A}$ and $\mathrm{B}$ received Dynamic Neuromuscular Stabilization exercises include 6 positions Prone static, Quadruped static, Quadruped dynamic, Bear position, Side sitting with dominant arm support, Side sitting with non-dominant arm support. Exercise in one position took approximately 
30 to 120 seconds. One exercise session took approximately 30 minutes, including 12 minutes of rest between each position. Both experimental group and control group also received conventional therapy in form of stretching and active assisted exercises, reaching activities, pegboard activity, gripping activity. Both the groups received intervention for 5 times a week for duration of 4 weeks. Assessment was done at the beginning and at the end of 4 weeks using Upper Extremity Function Index, grip strength and scapular abduction.

\section{OBSERVATION AND STATISTICAL} ANALYSIS

\section{FLOW DIAGRAM}

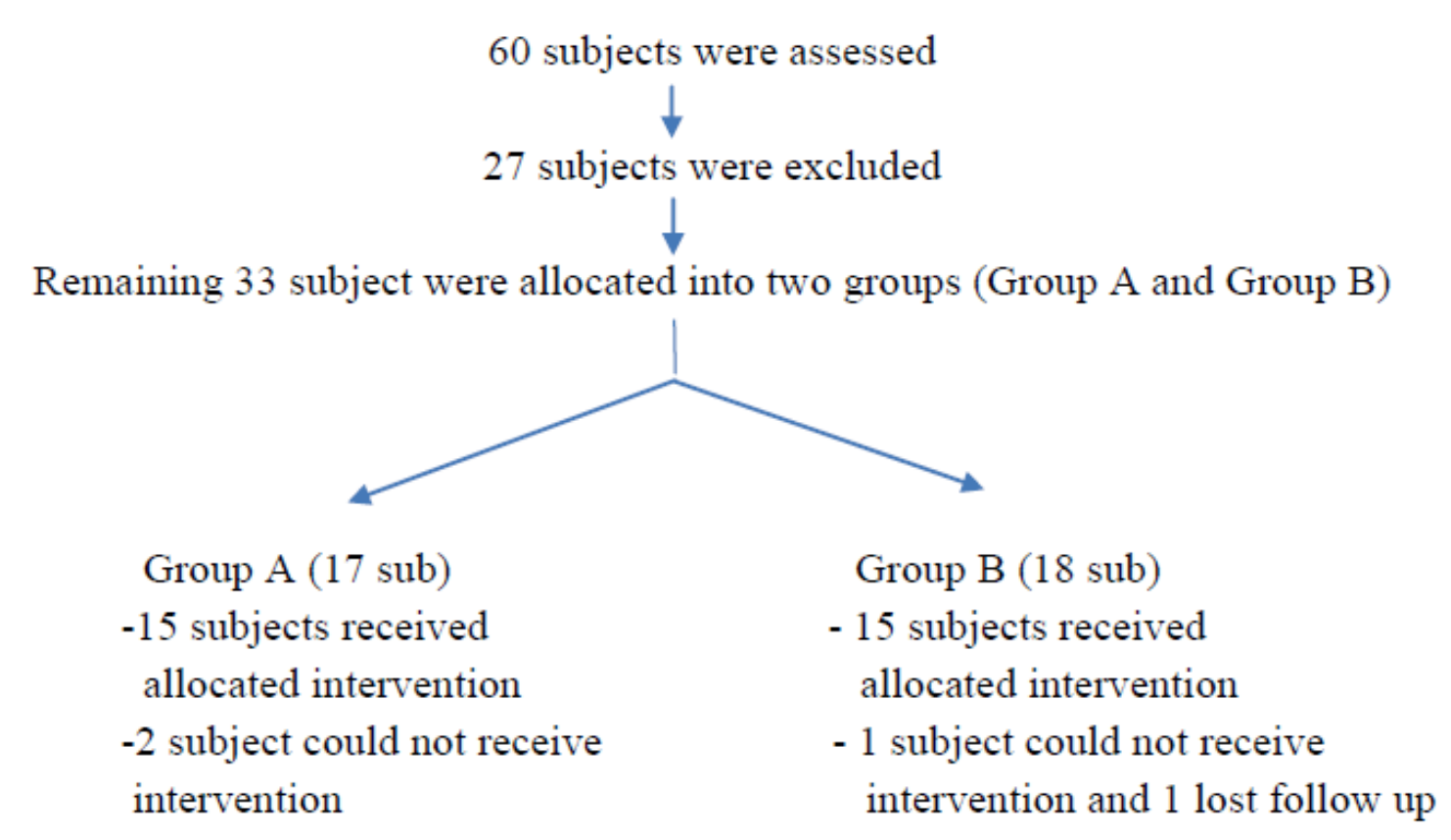

Remaining 33 subject were allocated into two groups (Group A and Group B)

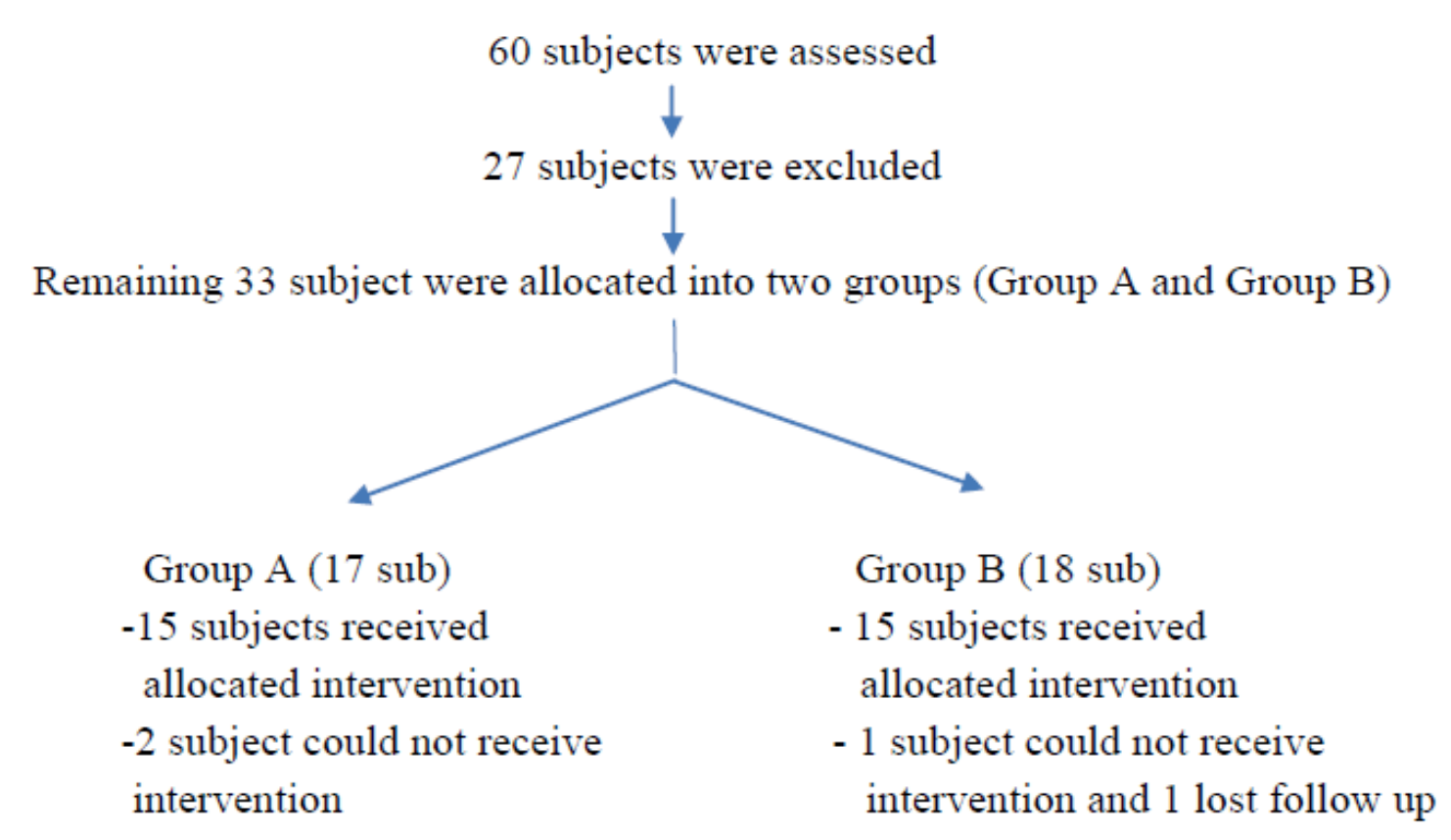

Group A (17 sub)

-15 subjects received

allocated intervention

-2 subject could not receive intervention
Data was initially explored to find out normal distribution using WinPepi software. Shapiro Wilk test was used for normal distribution analysis. For normally distributed data, paired t-test was used for analysis within same group and t-test was used for analysis between two different groups. Whereas for the data which was not normally distributed, Wilcoxon singed rank test was used for analysis within same group and Mann-Whitney singed rank test was used for analysis between two different groups. Statistical descriptive analysis was done by using Primer of Biostatistics software. Level of significance was set at 0.05 .

\section{RESULT}

After analysing pre and post scores of Upper Extremity Function Index, Grip Strength and Scapular Abduction (Table 1), the significant improvement was seen within the scores of both the groups post intervention with highly significant $p$ value $<0.0001$. After analysing mean values of the difference (post-pre) of Upper Extremity Function Index, Grip Strength and Scapular
Abduction in both the groups $\mathrm{A}$ and $\mathrm{B}$ (Table 2), it was observed that there was a significant improvement in scores of Upper Extremity Function index and Grip Strength in experimental group than control group. And it also showed that there was no significant difference between the mean values of difference (post-pre) of scapular abduction in experimental group and control group. 
Rutuja Jadhav et.al. Effect of scapular kinesiotaping as an adjunct to dynamic neuromuscular stabilization exercises on upper extremity functions in stroke patients.

Table 1: Comparison of pre and post interventional scores of upper extremity function index, grip strength and scapular abduction of experimental group and control group

\begin{tabular}{|l|l|l|l|l|l|l|l|}
\hline Outcome measure & Groups & \multicolumn{2}{l|}{ Pre-interventional score } & \multicolumn{2}{l|}{ Post-interventional score } & \multirow{2}{*}{ t-value } & P value \\
\cline { 3 - 7 } & & Mean & SD & Mean & SD & \\
\hline \multirow{2}{*}{ Upper Extremity Function Index } & Experimental group & 3.53 & 3.72 & 36.13 & 6.73 & 16.96 & $<0.0001$ \\
\cline { 2 - 7 } & Control group & 2.93 & 2.84 & 22.47 & 4.30 & 14.30 & $<0.0001$ \\
\hline \multirow{2}{*}{ Grip Strength } & Experimental group & 21.33 & 1.49 & 35.2 & 3.25 & 15.15 & $<0.0001$ \\
\cline { 2 - 8 } & Control group & 21.4 & 1.45 & 29.87 & 3.02 & 11.50 & $<0.0001$ \\
\hline \multirow{2}{*}{ Scapular Abduction } & Experimental group & 276.6 & 26.47 & 220.3 & 31.52 & 8.66 & $<0.0001$ \\
\cline { 2 - 8 } & Control group & 270.1 & 17.71 & 206.1 & 24.61 & 6.69 & $<0.0001$ \\
\hline
\end{tabular}

Table 2: Comparison of difference of mean (post-pre) of Upper Extremity Function Index, Grip Strength, and Scapular Abduction in experimental group and control group

\begin{tabular}{|c|c|c|c|c|c|c|}
\hline Outcome measure & Groups & Mean difference & SD & t value & Degree of freedom & P value \\
\hline \multirow[t]{2}{*}{ Upper Extremity Function Index } & Experimental group & 32.6 & 7.44 & \multirow[t]{2}{*}{5.542} & \multirow[t]{2}{*}{28} & \multirow[t]{2}{*}{$<0.0001$} \\
\hline & Control group & 19.53 & 5.29 & & & \\
\hline \multirow[t]{2}{*}{ Grip Strength } & Experimental group & 13.87 & 3.54 & \multirow[t]{2}{*}{4.599} & \multirow[t]{2}{*}{28} & \multirow[t]{2}{*}{$<0.0001$} \\
\hline & Control group & 8.46 & 2.85 & & & \\
\hline \multirow[t]{2}{*}{ Scapular Abduction } & Experimental group & 56.33 & 25.18 & \multirow[t]{2}{*}{0.663} & \multirow[t]{2}{*}{28} & \multirow[t]{2}{*}{0.513} \\
\hline & Control group & 64 & 37.01 & & & \\
\hline
\end{tabular}

\section{DISCUSSION}

Patients diagnosed with the stroke often present with a combination of muscle weakness and muscle imbalance. It leads to many impairments among which most common chronic impairment related to limitations of daily activities, functional activities and social roles is upper limb paresis. This makes the patient dependant on other person which compromises patient's quality of life. Therefore, the major goals of stroke rehabilitation include relearning of motor skills and restoring functions of upper extremities. Scapular stabilizers strengthening is especially important. ${ }^{[17]}$ Now a days, there are many new techniques are available for upper limb rehabilitation.

The present study assessed an added effect of scapular kinesiotaping as an adjunct to Dynamic Neuromuscular Stabilization Exercises along with conventional exercises on upper extremity functions in stroke patients.

Upper extremity functions in these subjects were assessed using three outcome measures; those are Upper Extremity Function Index, Grip strength by Aneroid manometer and scapular abduction by $\mathrm{MB}$ ruler. The results of this study showed that scapular Kinesio taping as an adjunct to dynamic neuromuscular stabilization exercises along with conventional exercises is beneficial in improving upper extremity functions in stroke patients at the end of 4 weeks.

In this study, both the groups showed improvement in upper extremity function index, grip strength and reduction in scapular abduction. However, when the scores between the group A and group B subjects undergoing scapular kinesiotaping as an adjunct to dynamic neuromuscular stabilization exercises and dynamic neuromuscular stabilization exercises along with conventional exercises respectively, were compared, they showed significant improvement of upper extremity functions and grip strength between both the groups. But group A subjects showed more significant improvement post treatment than group B.

After analysing pre and post scores of Upper Extremity Function Index (Table 1 ), the significant improvement was seen within the scores of both the groups post intervention with highly significant $\mathrm{p}$ value $<0.0001$.

Recovery from upper extremity impairments and activity limitations occurs mainly in the first 2-3 months after stroke. ${ }^{[18]}$ In stroke patients, as upper extremities get more affected, the muscles and the bony alignments are the predisposing factors for this. The major reason for the instability of an upper extremity is scapular malalignment. In these subjects, abnormal scapular and humeral movement patterns are present. Reduced 
voluntary neural drive after the stroke may disrupt the timing and activation of the scapulothoracic and rotator cuff muscles. During an exercise, as the arm is moved, the exercise may push the humerus into elevation angles higher than subject can actively produce without an assistance. This disturbs the normal upper extremity range of motion. ${ }^{[17]}$

Position of scapula is very important in functioning of upper limb. There is a hyperactivity of the upper trapezius and weakness or paralysis of serratus anterior and rhomboids muscles which are the main stabilizers of scapula. Scapular muscle weakness causes alteration in the position of the scapula resulting in altered biomechanics of upper limb which leads to inability of shoulder to go into overhead flexion and abduction.

The Dynamic Neuromuscular Stabilization Exercises played an important role in maintaining the normal alignment of the scapula by strengthening of the weak scapular stabilizers. Some studies reported that scapulothoracic muscles plays an important role in the stability of the shoulder complex and this stability is necessary to achieve an appropriate range of motion in the shoulder joint during arm elevation, as well as in the distal joints mobility such as wrist and fingers. ${ }^{[17,19]}$ Mobility of the upper limbs is crucial for daily living activities. ${ }^{10}$ Mandalidis and $\mathrm{O}$ ' Brien reported that when the proximal joints are efficiently stabilized by the surrounding musculature then only it is possible to have an efficient movement and proper range of motion of the muscles that act on a distal joints. ${ }^{[20]}$ This is achieved by Dynamic Neuromuscular Stabilization Exercises.

After analysing pre and post scores (Table 1) of Grip Strength, the significant improvement is seen within the scores of both the groups post intervention with highly significant p value $<0.0001$.

Veeger and van der Helm discussed that hand movement is dependent on three dimensional mobility of the shoulder complex, which in turn is dependent on co- ordination of trunk muscle. Pectoralis major and latissimus dorsi directly transfer arm forces to the thorax, serratus anterior and rhomboids press the scapula on thorax and thus provides stability. ${ }^{[21]} \mathrm{A}$ study done by Jeongok Yang found that there was improvement in the grip strength in the patients with protracted shoulder which suggests that positioning of scapula is very important for upper limb functions. ${ }^{[4]}$ Another study carried out by Geraldo Fabiano de Souza Moraes assessed Correlation among scapular positioning, functional postural analysis and upper extremities disability degree in sonographers found significant change in hand function when scapular position was altered. ${ }^{[22]}$

$$
\text { Dynamic }
$$

Neuromuscular

Stabilization approach compares the individual's stabilizing pattern with the stabilization developmental pattern of a healthy infant. By this technique, brain got stimulated and trained to automatically activate optimal movement patterns. Through these patterns co-activation of the stabilizers occurred, which resulted in improving proximal stability that improved grip strength of the patient. ${ }^{[12]}$

After analysing pre and post scores (Table 1) of Scapular Abduction, the significant improvement is seen within the scores of both the groups post intervention with highly significant $\mathrm{p}$ value $<0.0001$.

Scapular kinesiotaping has improved the alignment of scapula by inhibiting the over activity of the upper trapezius and facilitating the weak serratus anterior and rhomboids. Tape used for upper trapezius was in 'I' shape from insertion to origin to inhibit the over activity of it and for serratus anterior, tape used was in ' $\mathrm{I}$ ' shape and for rhomboids, tape used was in ' $\mathrm{X}$ ' shape from origin to insertion to facilitate the muscle contraction. In flaccid muscles, serratus anterior and rhomboids, the kinesiotape has exerted the tension via the restoring force in the direction of origin to the fixed base and thus it displaced the skin in the same direction. This brought about the support of 
the muscle contractions of both the muscles. In spastic upper trapezius muscle, the Kinesiotapes has exerted the tension in the direction of insertion to the fixed base and likewise displaced the skin in the same direction. That resulted in reduction in muscle contraction of upper trapezius muscle. ${ }^{[23]}$

The changes in scapular position occurred by altering the length- tension properties of these muscles and providing kinaesthetic awareness of scapular alignment during rest and movement of upper extremity. ${ }^{[11]}$ Kinesiotaping also produces traction and pressure on the skin which provides the cutaneous and sensory cues, thus providing additional proprioceptive input to the central nervous system, which helps in orientation of the scapula. ${ }^{[11]}$

After analysing mean values of the difference (post-pre) of Upper Extremity Function Index, Grip Strength and Scapular Abduction in both the groups $\mathrm{A}$ and $\mathrm{B}$ (Table 2), it was observed that there was a significant improvement in scores of Upper Extremity Function index and Grip Strength in group A than group B.

In group A subjects activation and strengthening of the weak scapular muscles by kinesiotaping and DNSE respectively, improved upper extremity functions, grip strength.

Proprioception serves to orient the body in space. Through the mechanoreceptors, we sense the position and movement of our joints. The sensors are present in the muscles, joints, tendons and skin. In this study, the proprioceptors present in the scapular muscles and skin over it got stimulated by Kinesiotapes and improved the awareness of proper positioning of the scapula. ${ }^{[23]}$

\section{Dynamic}

Neuromuscular

Stabilization Exercises mainly work on a principle of developmental kinesiology and maturing locomotor system. It compares every individual's stabilizing pattern to that of new-born. ${ }^{[12]}$ This concept says that, stabilizers have to work efficiently in order to have a proper functioning of the upper extremities. The Dynamic Neuromuscular Stabilization Exercises emphasize on focusing more on scapular muscles for efficiency of upper extremity which is same as that of a new-born's stabilizing pattern. ${ }^{[12]}$

And it also showed that there was no significant difference between the mean values of difference (post-pre) of scapular abduction in group A and group B. This showed that both the groups showed a significant improvement in scapular alignment by reduction of scapular abduction.

In upper extremity functions, the scapula provides a stable base from which glenohumeral mobility occurs. Scapula lies on the dorsal aspect of the ribcage from ribs $2^{\text {nd }}$ to $7^{\text {th }}$ and surrounded by a number of muscles acting as a stabilizer making it a supporting structure for upper extremity functions. ${ }^{[24]}$

The scapula is only attached to the thorax by ligamentous attachments at the acromioclavicular joint and by the muscular attachment of the serratus anterior and subscapularis. This muscular support holds the scapula in close proximity to the thorax and allows it to glide during movements of joint. While many muscles serve to stabilize the scapula, the main stabilizers are the serratus anterior, rhomboids, levator scapulae and trapezii. Weakness of scapular muscles leads to abnormal positioning of the scapula, disturbances in scapulohumeral rhythm and generalized shoulder complex dysfunction. Stability of the scapulothoracic joint depends on co-ordinated activity of the surrounding musculature. This joint is one of the least congruent joints in the body. No actual bony articulation exists between the scapula and thorax, which allow tremendous mobility in many directions. ${ }^{[24,25]}$

A study done on The Role of the Scapula by Russ Paine and Michael L. Voight, concluded that the lack of an actual bony articulation in the scapulothoracic region predisposes it to pathologic movement, rendering the glenohumeral joint 
highly dependent on its for stability and normal motion. ${ }^{[24]}$ There are many scapular muscles, out of which Serratus Anterior, Upper Trapezius and Rhomboids are more important in maintaining the position of scapula. ${ }^{[24]}$ Scapular position is maintained through force couples working in different directions. As scapular taping was applied to only upper trapezius, rhomboids and serratus anterior muscles for 4 weeks, it could not significantly bring about alteration in scapular position.

\section{CONCLUSION}

The present study concludes that Scapular Kinesiotaping as an adjunct to Dynamic Neuromuscular Stabilization Exercises is significantly effective to improve upper extremity functions and grip strength in post stroke patients, but less effective to change scapular position.

\section{Limitations}

1. All scapular movements were not considered.

2. No differentiation between particular sides of the hemiplegia was considered.

3. No long term follow up of the patients were taken.

\section{Clinical Implication}

Scapular Kinesiotaping as an adjunct to Dynamic Neuromuscular Stabilization Exercises along with conventional exercises can be given in order to improve upper extremity functions in stroke patients.

Abbreviations: Dynamic Neuromuscular Stabilization Exercises (DNSE), Upper Extremity Function Index (UEFI), Markus Bader Ruler (MB RULER), Middle Cerebral Artery (MCA), Central Nervous System (CNS), Montreal Cognitive Assessment (MOCA)

\section{ACKNOWLEDGEMENT}

I am honoured to thank my principal and my guide for giving me the opportunity to conduct this study and for always being supportive. Her guidance, help and encouragement was precious to understand my project better. A special thanks to all the staff members for their advice and opinions during the process of dissertation writing. I sincerely thank to all the participants for their active and sincere participation in my study. I would like to take an opportunity to thank my colleagues who were there when needed. I am heartily thankful to all.

\section{Conflict of Interest: None}

\section{Source of Funding: None}

\section{Ethical Approval: Approved}

\section{REFERENCES}

1. O' Sullivan SBO, Schmitz TJ. Textbook of physical rehabilitation. $5^{\text {th }}$ edition. Rehabilitation 2007 471-474 p.

2. Pandian J. et al. Stroke Epidemiology and stroke care services in India. J Stroke 2013;15(3):128-134

3. Lai SM, et al. Persisting consequences of stroke measured by the stroke impact scale. Stroke2002; 33:1840-4.

4. Yang J, Lee B, Jeon S, Han B, Han D. The effects of active scapular protraction on the Muscle Activation and Function of the Upper Extremity. J Phys Ther Sci [Internet]. 2014;26(4):599-603

5. Kim Y. The effects of EMG-triggered functional electrical stimulation on upper extremity function in stroke patients. Kor Acad Phys Ther Rehabil Sci 2013; 2(1):1-6.

6. Ajit Dabholkar, Devanshi Mehta, Sujata Yardi, Tejashree Dabholkar: Assessment of scapular behaviour in stroke patients. Int $\mathrm{J}$ Health Rehabil Sci.2015; 4(2): 95-102

7. De Kroon JR, et al.: Therapeutic electrical stimulation to improve motor control and functional abilities of the upper extremity after stroke: a systematic review clinical rehabilitation. Clin Rehabil. 2002;16:350-60

8. Orbetta D, et al. Constrained induced movement therapy in stroke patients: systematic review and meta-analysis, Eur j Phys Rehabil Med 2010;46:537-44

9. Masiero S, Celia A, Rosati G, Armani M. Robotic-assisted rehabilitation of the upper limb after acute stroke. Arch Phys Med Rehabil. 2007 Feb;88(2):142-9. doi: 
Rutuja Jadhav et.al. Effect of scapular kinesiotaping as an adjunct to dynamic neuromuscular stabilization exercises on upper extremity functions in stroke patients.

10.1016/j.apmr.2006.10.032.

PMID: 17270510.

10. Chiang-soon Song, PhD, OT: Effects of scapular stabilization exercises on function of paretic upper extremity of chronic stroke patients. J Phys Ther Sci. 2013; 25(4):403405

11. Dhwanit shah, et.al Comparative study of the effect of kinesiotaping on scapular stability and upper limb function in recovering hemiplegics with scapular weakness. Chron Youn Sci. 2013; vol 4(2): 121-129

12. Dynamic Neuromuscular Stabilization (DNS): According to Kolar A Developmental Kinesiology Approach What is DNS?

13. Kobesova A, Dzvonik J, Kolar P, Sardina A, Andel R. Effects of shoulder girdle dynamic stabilization exercise on hand muscle strength. Isokinet Exerc Sci. 2015; 23 (1):21-32.

14. Ewa Jaraczewska and Carol Long Kinesiotaping in stroke: improving functional use of the upper extremity in hemiplegia by Ewa Jaraczewska and Carol Long, Top Stroke Rehabil 2006; 13(3): 3142

15. Kenzo Kase, D.C. Illustrated Kinesio taping. $4^{\text {th }}$ edition.

16. Yasukawa A, Patel P, Sisung C. The functional effects of Kinesiotaping in an acute pediatric rehabilitation setting as measured by the Melbourne Assessment. Paper presented at the Kinesiotaping Association Symposium. Am J Occup Ther. 2006; 60(1): 104-10.

17. Hardwick DD, Lang CE. Scapular and humeral movement patterns of people with stroke during range of motion exercises. $\mathrm{J}$ Neurol Phys Ther 2011;35(1):18-25
18. De Baet's L et.al. Scapulohumeral control after stroke: A preliminary study of test retest reliability and discriminant validity of clinical scapular protocol (ClinScap) NeuroRehabilitation. 2016; 38(4):359-70.

19. Nascimento LR et al. Isometric hand grip strength correlated with isokinetic data of the shoulder stabilizers in individuals with chronic stroke. . J Bodyw Mov Ther, 2012;16:275-280

20. Mandalidis D, O’brien M. relationship between hand grip isometric strength and isokinetic moment data of the shoulder stabilizers. J Bodyw Mov Ther, 2010;14: 19-26

21. Veeger HE, van der Helm FC. Shoulder function: the perfect compromise between mobility and stability. J Biomech. 2007; 40(10):2119-29. doi: 10.1016/j.jbiomech.2006.10.016. Epub 2007 Jan 12. PMID: 17222853.

22. Geraldo Fabiano de Souza Moraes et al. Correlation among scapular positioning, functional postural analysis and upper extremities disability degree in sonographers (DASH Brazil). Time 2009; 42(3):31-6

23. Kumbrink B. K Taping: An Illustrated Guide. 2012

24. Paine RM, Voight $M$ The Role of the Scapula. J Orthop Sport Phys Ther. 1993;18(1):386-91

25. Now B. Role of correct scapula setting with major exercises.2017;1-4

How to cite this article: Jadhav R, Pazare S. Effect of scapular kinesiotaping as an adjunct to dynamic neuromuscular stabilization exercises on upper extremity functions in stroke patients. Int J Health Sci Res. 2022; 12(1):13-22. DOI: https://doi.org/10.52403/ijhsr.20220103 